\title{
Riluzole enhances the antitumor effects of temozolomide via suppression of MGMT expression in glioblastoma
}

\author{
Tetsuya Yamada, MD, 1,2 Shohei Tsuji, BS, ${ }^{1}$ Shinsuke Nakamura, PhD, ${ }^{1}$ Yusuke Egashira, MD, PhD, ${ }^{2}$ \\ Masamitsu Shimazawa, PhD, ${ }^{1}$ Noriyuki Nakayama, MD, PhD, ${ }^{2}$ Hirohito Yano, MD, PhD, ${ }^{2}$ \\ Toru Iwama, MD, PhD, ${ }^{2}$ and Hideaki Hara, PhD ${ }^{1}$ \\ ${ }^{1}$ Molecular Pharmacology, Department of Biofunctional Evaluation, Gifu Pharmaceutical University; and 2Department of \\ Neurosurgery, Gifu University Graduate School of Medicine, Gifu, Japan
}

OBJECTIVE Glutamatergic signaling significantly promotes proliferation, migration, and invasion in glioblastoma (GBM). Riluzole, a metabotropic glutamate receptor 1 inhibitor, reportedly suppresses GBM growth. However, the effects of combining riluzole with the primary GBM chemotherapeutic agent, temozolomide (TMZ), are unknown. This study aimed to investigate the efficacy of combinatorial therapy with TMZ/riluzole for GBM in vitro and in vivo.

METHODS Three GBM cell lines, T98G (human; $0^{6}$-methylguanine DNA methyltransferase [MGMT] positive), U87MG (human; MGMT negative), and GL261 (murine; MGMT positive), were treated with TMZ, riluzole, or a combination of both. The authors performed cell viability assays, followed by isobologram analysis, to evaluate the effects of combinatorial treatment for each GBM cell line. They tested the effect of riluzole on MGMT, a DNA repair enzyme causing chemoresistance to TMZ, through quantitative real-time reverse transcription polymerase chain reaction in T98G cells. Furthermore, they evaluated the efficacy of combinatorial TMZ/riluzole treatment in an orthotopic mouse allograft model of MGMT-positive GBM using C57BL/6 J mice and GL261 cells.

RESULTS Riluzole displayed significant time- and dose-dependent growth-inhibitory effects on all GBM cell lines assessed independently. Riluzole enhanced the antitumor effect of TMZ synergistically in MGMT-positive but not in MGMTnegative GBM cell lines. Riluzole singularly suppressed MGMT expression, and it significantly suppressed TMZ-induced MGMT upregulation $(p<0.01)$. Furthermore, combinatorial TMZ/riluzole treatment significantly suppressed tumor growth in the intracranial MGMT-positive GBM model $(p<0.05)$.

CONCLUSIONS Riluzole attenuates TMZ-induced MGMT upregulation and enhances the antitumor effect of TMZ in MGMT-positive GBMs. Therefore, combinatorial TMZ/riluzole treatment is a potentially promising novel therapeutic regimen for MGMT-positive GBMs.

https://thejns.org/doi/abs/10.3171/2019.12.JNS192682

KEYWORDS glioblastoma; MGMT; riluzole; temozolomide; combinatorial therapy; oncology

$\mathrm{G}$ LIOBLASTOMA (GBM) is the most aggressive and one of the most common tumors of the central nervous system. Despite current combinatorial treatment modalities, including resection, radiation therapy, and chemotherapy, the prognosis for patients with GBM remains poor owing to the tumor's heterogeneity, high proliferation, and invasiveness. ${ }^{29}$ Temozolomide (TMZ), an alkylating agent capable of crossing the bloodbrain barrier, is almost the only chemotherapeutic agent used to treat GBM and has been reportedly highly effective in some cases. ${ }^{12}$ However, chemoresistance to alkyl- ating agents including TMZ frequently occurs owing to the effect of $\mathrm{O}^{6}$-methylguanine DNA methyltransferase (MGMT), a DNA repair enzyme upregulated in cancers. MGMT inhibits the effects of TMZ by eliminating the TMZ-induced alkylation adducts from the $\mathrm{O}^{6}$ position in guanine. ${ }^{18}$ MGMT-positive GBM cells, which are GBM cells with unmethylated MGMT promoters, exhibit chemoresistance to TMZ through this repair mechanism. This highlights the need for additional effective chemotherapeutics for GBM that are potentially applicable for increasing the efficacy of TMZ, perhaps by targeting

ABBREVIATIONS CCK-8 = Cell Counting Kit-8; Cl = combination index; DMEM = Dulbecco's modified Eagle medium; DMSO = dimethyl sulfoxide; FBS = fetal bovine serum; GBM = glioblastoma; $I_{2}=20 \%$ inhibitory concentration; IFN- $\beta=$ interferon- $\beta$; MGMT = $0^{6}$-methylguanine DNA methyltransferase; RT-PCR = reverse transcription polymerase chain reaction; $\mathrm{TMZ}=$ temozolomide.

SUBMITTED October 1, 2019. ACCEPTED December 30, 2019.

INCLUDE WHEN CITING Published online March 13, 2020; DOI: 10.3171/2019.12.JNS192682. 
MGMT. Concurrent administration of the small nucleoside inhibitor, $\mathrm{O}^{6}$-benzylguanine, and $\mathrm{TMZ}$ was previously reported in a clinical trial to restore chemosensitivity in patients with recurrent TMZ-resistant malignant glioma by mimicking the target of MGMT. ${ }^{23}$ Unfortunately, however, TMZ/O ${ }^{6}$-benzylguanine combination therapy was also found to cause severe myelosuppression. ${ }^{23}$ Despite several clinical trials assessing the impact of TMZ combined with other drugs, none of the combinations tested so far have prolonged the overall survival of GBM patients in comparison with TMZ administration alone. ${ }^{7,13}$

Glutamate is one of the major excitatory neurotransmitters regulating neuronal and synaptic activity, and it plays an important role in activating proliferation, migration, and invasion among GBM cells..$^{10}$ Glutamate activates not only the ionotropic glutamate receptors (iGluRs), such as the $\alpha$-amino-3-hydroxy-5-methyl-4-isoxazole propionate receptors (AMPARs), $N$-methyl-D-aspartate receptors (NMDARs), and kainic acid receptors (KARs), but also the G-protein-coupled metabotropic glutamate receptors (mGluRs), a family containing eight subtypes (mGluR1 to mGluR8). ${ }^{36}$ Riluzole is a selective mGluR1 antagonist of the benzothiazole class, approved by the U.S. FDA for treating amyotrophic lateral sclerosis. ${ }^{28,36}$ Moreover, riluzole reportedly inhibits glutamate release via inactivation of voltage-dependent sodium channels and serves as a noncompetitive antagonist of NMDARs. ${ }^{11}$ Recent studies have reported that riluzole inhibits GBM progression by suppressing the PI3K/AKT/mTOR pathway. ${ }^{36}$ Although riluzole monotherapy reportedly did not improve the survival of rats transplanted with GBM, it enhanced their sensitivity to radiotherapy. ${ }^{33}$ However, no studies have investigated the efficacy of combinatorial therapy with TMZ and riluzole for GBM, nor has there been any evidence regarding the effectiveness of riluzole against MGMT-induced chemoresistance.

This study aimed to evaluate the effect of riluzole on MGMT expression and assess the effectiveness of combinatorial treatment with TMZ and riluzole against GBM in vitro and in vivo.

\section{Methods \\ Cell Culture}

We used the human GBM cell lines T98G and U87MG and one murine GBM cell line, GL261. T98G cells were purchased from the JCRB Cell Bank, and U87MG cells were from the American Type Culture Collection. GL261 cells were kindly provided by Dr. Masanao Saio (Gunma University, Japan).

As previously described, ${ }^{21}$ T98G, U87MG, and GL261 cells were cultured in Dulbecco's modified Eagle medium (DMEM) with low glucose (Nacalai Tesque), supplemented with $10 \%$ fetal bovine serum (FBS; Valeant), $100 \mathrm{U} / \mathrm{mL}$ penicillin, and $100 \mu \mathrm{g} / \mathrm{ml}$ streptomycin in $10-\mathrm{cm}$ dishes (BD Biosciences) at $37^{\circ} \mathrm{C}$ in $5 \% \mathrm{CO}_{2}$.

\section{Cell Viability Assay}

Cell Counting Kit-8 (CCK-8) cell viability assays were conducted as previously described. ${ }^{21}$ T98G, U87MG, and GL261 cells were seeded in a 96-well plate $\left(2.0 \times 10^{3}\right.$ cells/ well) with culture medium supplemented with $10 \%$ FBS. After 24 hours of incubation, the medium was changed to DMEM supplemented with $10 \%$ FBS and the drugs in accordance with the following 4 conditional groups: notreatment control (dimethyl sulfoxide [DMSO]), TMZ (10, $20,30,40,50,60,100,150,200,300,400,500$, or 600 $\mu \mathrm{M})$, riluzole $(1,2,3,4,5,10,15,20,25,30$, or $35 \mu \mathrm{M})$, and the combinatorial treatment (TMZ and riluzole). After 24,48 , or 72 hours of treatment, CCK- 8 assays were performed to determine cell viabilities. The absorbance of each well was measured at $450 \mathrm{~nm}$ with a reference wavelength of $660 \mathrm{~nm}$ using a microplate reader.

\section{Isobologram Analysis}

To determine whether riluzole has a synergistic or additive effect with TMZ on each cell line, we conducted isobologram analysis. We selected a $20 \%$ inhibitory concentration $\left(\mathrm{IC}_{20}\right)$ as the reference concentration, which is defined as the drug concentration reducing the cell viability of a treated group to $20 \%$ of the level of the no-treatment control group.

Based on the results of the cell viability assay, the $\mathrm{IC}_{20}$ of TMZ and riluzole was determined for each cell line. We evaluated the growth-inhibitory effects upon combinatorial treatment in comparison with the control group by measuring cell viabilities after 72 hours of treatment. Only the concentration combinations exhibiting 20\% growth-inhibitory effects were plotted on isobolograms.

On isobologram graphs, the points plotted below, on, or above the theoretical line of additivity indicate synergism, additivity, or antagonism of the drug combination, respectively. ${ }^{1}$ The combination index (CI) was determined using the following equation: $\mathrm{CI}=[\mathrm{C}] \mathrm{T}_{20} / \mathrm{IC}_{20} \mathrm{~T}+[\mathrm{C}] \mathrm{R}_{20} / \mathrm{IC}_{20} \mathrm{R}$. $[C] \mathrm{T}_{20}$ and $[\mathrm{C}] \mathrm{R}_{20}$ represent the concentrations of TMZ and riluzole, respectively, that exhibit $20 \%$ inhibition on cell growth upon combinatorial treatment. $\mathrm{CI}<1, \mathrm{CI}=1$, and $\mathrm{CI}>1$ indicate synergism, additivity, and antagonism of the drug combination, respectively. ${ }^{1}$

\section{Quantitative Real-Time Reverse Transcription Polymerase Chain Reaction Analysis}

To evaluate the effect of riluzole on the expression of $M G M T$ mRNA expression, we performed quantitative real-time reverse transcription polymerase chain reaction (RT-PCR) analysis. T98G cells were seeded in a 12-well plate $\left(2.0 \times 10^{4}\right.$ cells/well $)$ with DMEM supplemented with $10 \%$ FBS. After 24 hours of incubation, the medium was changed, and GBM cells were cultured under the following conditions for 48 hours: no-treatment control (DMSO), TMZ $(300 \mu \mathrm{M})$, riluzole $(1,5$, or $10 \mu \mathrm{M})$, or a combination of TMZ $(300 \mu \mathrm{M})$ and riluzole $(5 \mu \mathrm{M})$. After 48 hours of treatment, RNA was isolated from T98G cells using Nucleo Spin RNA II (Takara). RNA concentrations were determined using NanoVue Plus (GE Healthcare Japan). Single-strand cDNAs were synthesized from the isolated RNAs via reverse transcription with a PrimeScript RT Reagent Kit (Perfect Real Time; Takara). Quantitative realtime RT-PCR was performed using TB Green Premix Ex Taq II (Tli RNaseH Plus; Takara) and a TP800 Thermal Cycler Dice Real Time System (Takara). All aforemen- 
tioned procedures were carried out in accordance with the manufacturer's instructions. A human MGMT quantitative PCR primer pair was purchased from Sino Biological Inc. The PCR primer sequences for GAPDH (internal control) were as follows: 5'-TGT GTC CGT CGT GGA TCT GA-3' (forward) and 5'-TTG CTG TTG AAG TCG CAG GAG-3' (reverse). The cycling conditions were in accordance with the manufacturer's protocol. The results are expressed as relative gene expression levels normalized to that of GAPDH.

\section{Establishment of the Orthotopic Mouse Allograft Model of MGMT-Positive GBM}

All animal procedures were conducted in accordance with the ARRIVE (Animal Research: Reporting of In Vivo Experiments) guidelines and were approved by the animal experiment committee of Gifu Pharmaceutical University, Japan.

Eight-week-old male C57BL/6 J mice (Japan SLC, Ltd.) were used herein. All mice were housed at $24^{\circ} \mathrm{C}$ under a 12-hour light-dark cycle. On day 0 , the mice received orthotopic intracranial injections of $1.0 \times 10^{5} \mathrm{GL} 261$ cells in $2 \mu \mathrm{L}$ of PBS using a Hamilton microliter syringe placed in the left striatum at previously described coordinates ${ }^{21}$ : $1 \mathrm{~mm}$ anterior, $2 \mathrm{~mm}$ left lateral to the bregma, and $3 \mathrm{~mm}$ below the cranial surface. The mice were randomly divided into the no-treatment control group $(n=12)$ and treatment groups. The latter received TMZ on days 14-18 $(50 \mathrm{mg} / \mathrm{kg} / \mathrm{day})(\mathrm{n}=12)$, or a combination of riluzole on days 13-18 (15 mg/kg/day) and TMZ on days 14-18 (50 $\mathrm{mg} / \mathrm{kg} /$ day) $(\mathrm{n}=12)$. All drugs were orally administered. The dosages of TMZ and riluzole were based on previous reports. ${ }^{16,30,33}$ The mice were monitored daily during the experiment, and those displaying severe neurological deficits, severe pain, or anorexic symptoms were euthanized with pentobarbital.

On day 20, 10 mice in each group were deeply anesthetized and transcardially perfused with $4 \%$ paraformaldehyde (Wako). The brains were dissected out, fixed in $4 \%$ paraformaldehyde, and embedded in paraffin (Leica Biosystems). To determine the maximum cross-sectional areas and volumes of the tumors, samples were cut into 5- $\mu$ m-thick coronal sections, stained with $\mathrm{H} \& \mathrm{E}$, and photographed using an All-in-One Fluorescence Microscope (BZ-X710; Keyence).

\section{Statistical Analysis}

Each in vitro experiment was performed in triplicate. Data are presented as mean \pm SEM. Statistical analyses were performed using one-way ANOVA, followed by Dunnett's test or Tukey's test for post hoc analysis. Thompson's rejection tests were performed for individual values to eliminate outliers. $\mathrm{p}$ values $<0.05$ were considered statistically significant. All tests were conducted using the statistical software JMP 13 (SAS Institute Inc.).

\section{Results}

\section{Riluzole Independently Inhibits GBM Cell Proliferation}

We first evaluated the effects of riluzole on the growth of GBM cell lines in monolayer cultures. CCK-8 cell vi- ability assays were conducted on two MGMT-positive cell lines (T98G and GL261) and one MGMT-negative cell line (U87MG). ${ }^{6,37}$ When administered alone, riluzole (5, 10 , or $20 \mu \mathrm{M}$ ) had significant time- and dose-dependent inhibitory effects on the proliferation of all GBM cell lines tested herein (Fig. 1A-C). Cell viabilities were significantly lower in the treatment groups than in the no-treatment control group at the following riluzole concentrations and time points in each cell line: T98G cells after 48 and 72 hours of treatment with $20 \mu \mathrm{M}$ ( $\mathrm{p}<0.01)$; U87MG cells after 48 hours of treatment with $20 \mu \mathrm{M}(\mathrm{p}<0.01)$ and after 72 hours of treatment with 5,10 , and $20 \mu \mathrm{M}(\mathrm{p}<0.01)$; and GL261 cells after 24, 48, and 72 hours treatment with 20 $\mu \mathrm{M}(\mathrm{p}<0.01)$ and 48 hours of treatment with $5 \mu \mathrm{M}(\mathrm{p}<$ $0.05)$.

\section{Riluzole Sensitizes MGMT-Positive GBM Cells to TMZ}

We treated two MGMT-positive (unmethylated MGMT promoter) cell lines (T98G and GL261) and one MGMTnegative (methylated MGMT promoter) cell line (U87MG) with TMZ, riluzole, or a combination of the two for 72 hours and evaluated cell proliferation via CCK-8 cell viability assays (Fig. 2A-C). After 72 hours, TMZ exerted significant dose-dependent inhibitory effects on the proliferation of all GBM cell lines tested herein. Cell viabilities were significantly decreased at $400 \mu \mathrm{M}$ in T98G cells ( $\mathrm{p}$ $<0.01$ ), $20 \mu \mathrm{M}$ in U87MG cells ( $<<0.01$ ), and $50 \mu \mathrm{M}$ in GL261 cells ( $\mathrm{p}<0.05$ ), compared with the no-treatment control group. As described in Fig. 1, riluzole exerted significant dose-dependent inhibitory effects on all GBM cell lines tested herein after 72 hours of treatment. Cell viabilities were significantly decreased at $10 \mu \mathrm{M}$ in T98G cells $(\mathrm{p}<0.05), 5 \mu \mathrm{M}$ in U87MG cells $(\mathrm{p}<0.05)$, and $15 \mu \mathrm{M}$ in GL261 cells $(\mathrm{p}<0.01)$, compared with the no-treatment control. The effects of combinatorial therapy of TMZ and riluzole on GBM cell lines were evaluated at drug concentrations largely ineffective when administered alone. The results for each cell line are shown in Fig. 2 (A: T98G, B: U87MG, C: GL261). In T98G human MGMT-positive cells, the combination of $300 \mu \mathrm{M}$ TMZ and $5 \mu \mathrm{M}$ riluzole demonstrated a significant inhibitory effect on cell proliferation ( $p<0.01$; Fig. 2A). Furthermore, in GL261 murine MGMT-positive cells, the combination of $40 \mu \mathrm{M}$ TMZ and $5 \mu \mathrm{M}$ riluzole demonstrated a significant inhibitory effect ( $\mathrm{p}<0.01$; Fig. 2C). In contrast, in U87MG human MGMT-negative cells, the combination of $10 \mu \mathrm{M}$ TMZ and $3 \mu \mathrm{M}$ riluzole did not significantly inhibit cell proliferation (Fig. 2B).

Thereafter, we determined whether treatment with 5 $\mu \mathrm{M}$ riluzole enhanced the cytostatic effect of TMZ at other concentrations in T98G (Fig. 2D) and GL261 (Fig. 2E) cells. Both these cell lines were significantly sensitized to TMZ in the presence of $5 \mu \mathrm{M}$ riluzole at all TMZ concentrations tested.

\section{Riluzole Acts Synergistically With TMZ Against MGMT-Positive GBM Cells}

Further, we investigated whether riluzole treatment synergistically enhances the cytostatic effect of TMZ on each GBM cell line. Prior to this analysis, we preliminar- 

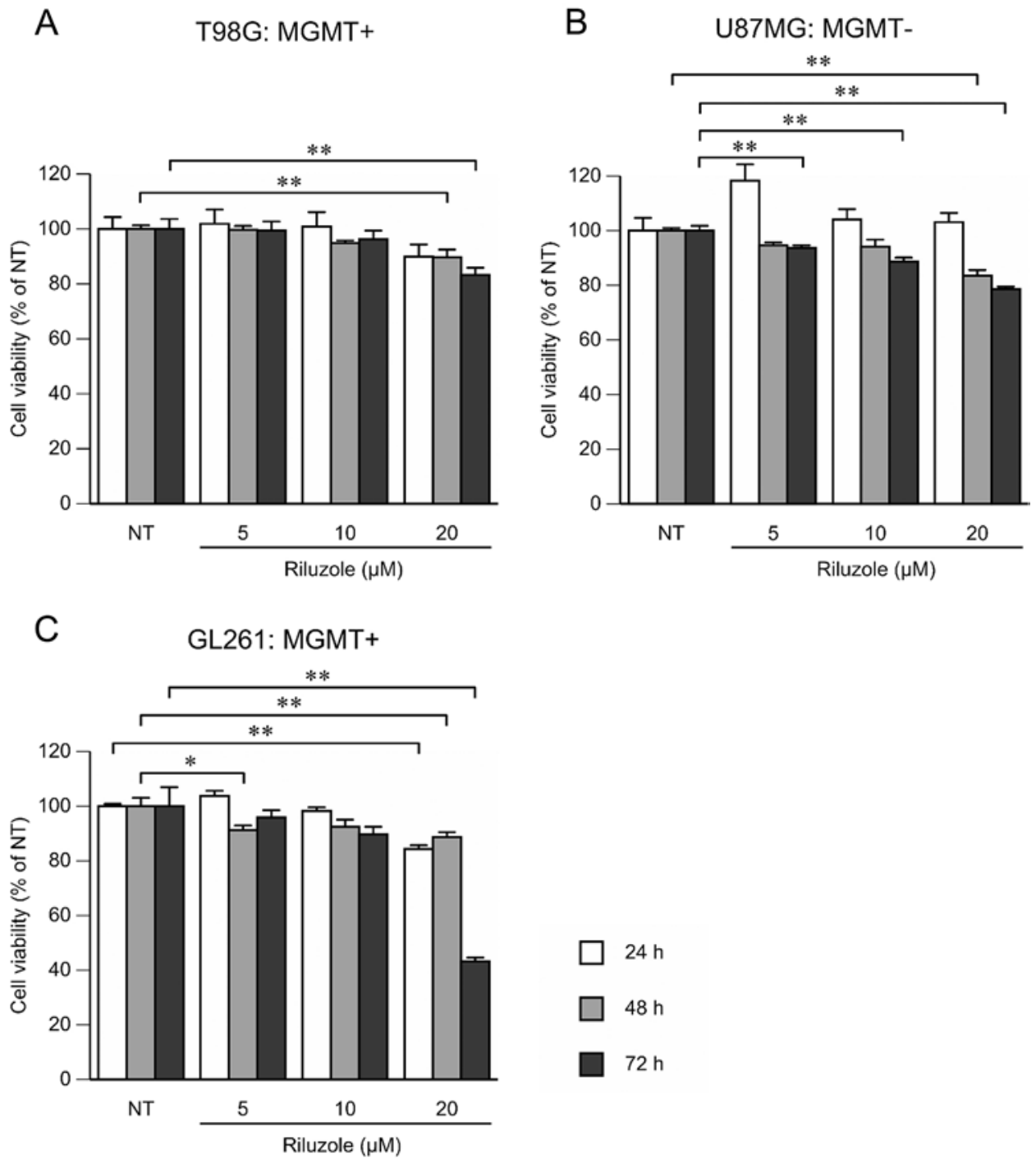

FIG. 1. The effect of riluzole on GBM cell growth. CCK-8 cell viability assays were performed on two MGMT-positive GBM cell lines (T98G, A, and GL261, C) and one MGMT-negative GBM cell line (U87MG, B) after 24, 48, and 72 hours (h) of treatment with riluzole at 3 different concentrations $(5,10$, and $20 \mu \mathrm{M})$. Each column and bar represents the mean \pm SEM $(n=6)$. * $p<0.05$ versus no treatment (NT); ${ }^{* *} p<0.01$ versus NT (one-way ANOVA followed by Dunnett's test).

ily determined the $\mathrm{IC}_{20}$ of $\mathrm{TMZ}$ (Fig. 3A) and riluzole (Fig. 3B) for each GBM cell line, using CCK-8 viability assays. In T98G, U87MG, and GL261 cells, the $\mathrm{IC}_{20}$ for TMZ was $481 \mu \mathrm{M}, 75 \mu \mathrm{M}$, and $170 \mu \mathrm{M}$, whereas the $\mathrm{IC}_{20}$ for riluzole was $16 \mu \mathrm{M}, 19 \mu \mathrm{M}$, and $13 \mu \mathrm{M}$, respectively. We then conducted isobologram analysis to determine whether the effects of combinatorial treatment with TMZ and riluzole were synergistic or additive (Fig. 3C-E). For T98G and GL261 cells, the isobologram analysis demonstrated clear synergism at all drug concentrations tested (Fig. 3C and E). In contrast, combinatorial treatment displayed additive or even antagonistic effects in U87MG cells (Fig. 3D). The concentration combinations used to construct isobolograms and determine the CIs are shown in Table 1. In T98G cells, the strongest synergism between TMZ and riluzole was observed with the combination of $96 \mu \mathrm{M}$ TMZ and $8 \mu \mathrm{M}$ riluzole, with a CI of 0.7. In
GL261 cells, the strongest synergism was observed with a combination of $34 \mu \mathrm{M}$ TMZ and $6 \mu \mathrm{M}$ riluzole, with a $\mathrm{CI}$ of 0.66 . In U87MG cells, the lowest $\mathrm{CI}$ was 0.97 , which was observed with a combination of $45 \mu \mathrm{M}$ TMZ and 7 $\mu \mathrm{M}$ riluzole, whereas the other combinations displayed antagonism.

\section{Riluzole Inhibits MGMT mRNA Expression in GBM Cells in a Dose-Dependent Manner}

To evaluate the effect of riluzole on $M G M T$ mRNA levels, we performed quantitative real-time RT-PCR analysis. In T98G MGMT-positive GBM cells, riluzole downregulated MGMT mRNA in a dose-dependent manner. MGMT mRNA levels differed significantly between the nontreated control and $10-\mu \mathrm{M}$ riluzole-treated cells after 48 hours ( $\mathrm{p}<0.05$; Fig. 4, left). 

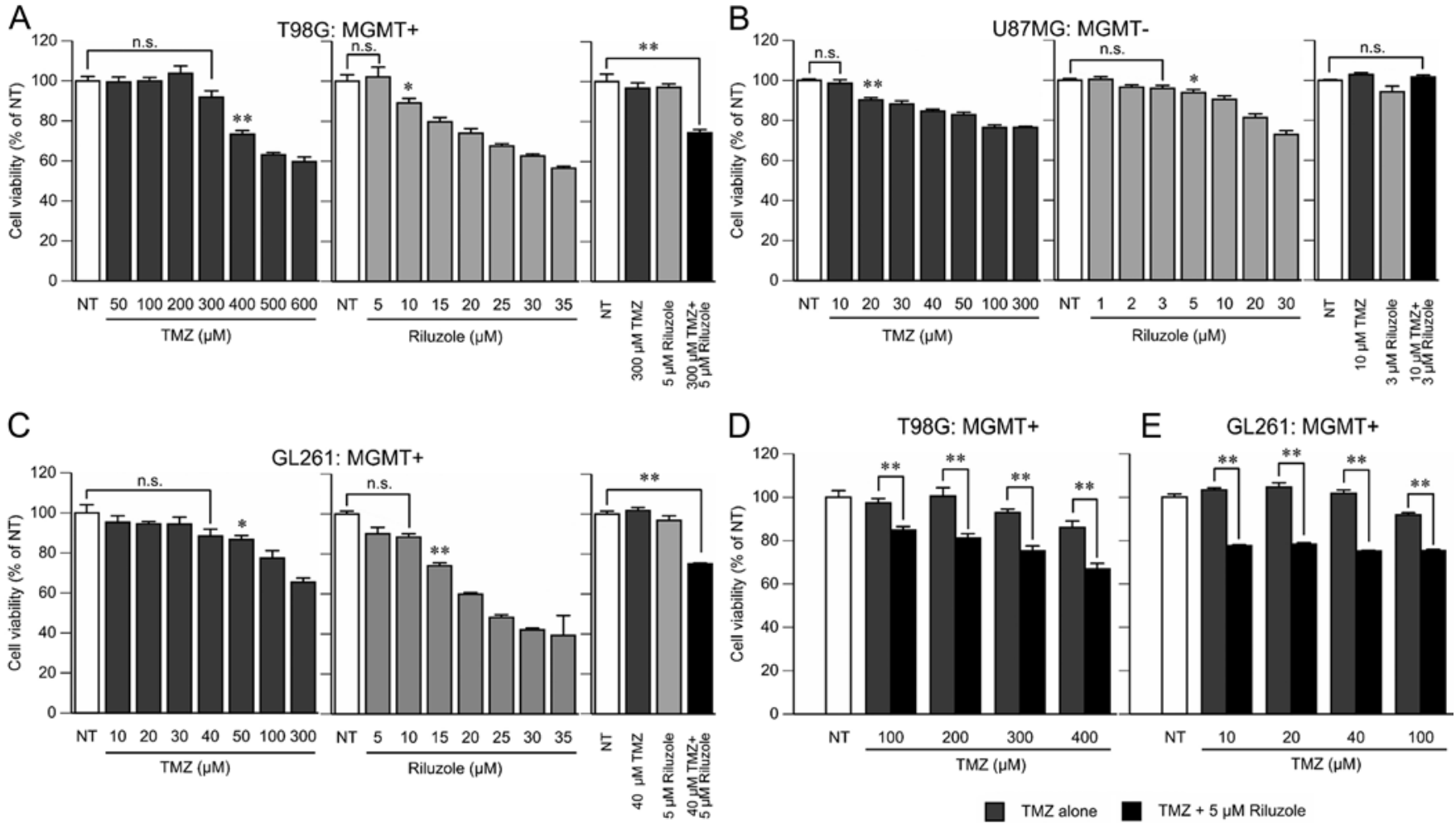

FIG. 2. The effect of combining riluzole with TMZ on the growth of the GBM cell lines T98G, U87MG, and GL261. CCK-8 cell viability assays were performed for two MGMT-positive GBM cell lines (T98G [A] and GL261 [C]) and one MGMT-negative GBM cell line (U87MG [B]) after 72 hours of treatment with TMZ, riluzole, or a combination of the two at concentrations that were not significantly effective during monotherapy. Each column and bar represents the mean \pm SEM $(n=6)$. ${ }^{*} p<0.05$ versus NT; ${ }^{* *} p<$ 0.01 versus NT. In two MGMT-positive GBM cell lines (T98G [D] and GL261 [E]), the efficacy of riluzole (5 $\mu$ M) combined with 4 different concentrations of TMZ was evaluated using CCK-8 assays after 72 hours of treatment. Each column and bar represents the mean \pm SEM $(n=6)$. ${ }^{* *} p<0.01$ versus TMZ alone (Student $t$-test). n.s. = not significant (one-way ANOVA followed by Dunnett's test).

\section{Riluzole Inhibits TMZ-Induced MGMT Expression in GBM Cells}

Quantitative real-time RT-PCR analysis revealed significant upregulation of MGMT mRNA in T98G cells upon treatment with $300 \mu \mathrm{M}$ TMZ for 48 hours $(\mathrm{p}<0.001$; Fig. 4 , right). We then investigated whether riluzole treatment decreases TMZ-induced upregulation of MGMT. Combinatorial treatment with $300 \mu \mathrm{M} \mathrm{TMZ}$ and $5 \mu \mathrm{M}$ riluzole for 48 hours significantly downregulated $M G M T$ mRNA in comparison with treatment with $300 \mu \mathrm{M}$ TMZ alone (p $<0.01$; Fig. 4, right).

\section{Riluzole Enhances the Inhibitory Effect of TMZ in an MGMT-Positive Mouse Model of Intracranial GBM}

We generated a mouse model of MGMT-positive GBM by injecting GL261 cells into the left striatum of adult mice. The protocol for drug administration is illustrated in Fig. 5A. In this study, two mice in each group were euthanized because of technical errors during drug administration or symptoms of anorexia following drug administration. To evaluate the effects of the drug therapies, we measured the maximum cross-sectional area and volume of the intracranial tumors after treatment (Fig. 5B-D). The $50-\mathrm{mg} / \mathrm{kg}$ dose of TMZ was selected because it is equivalent to the dose of maintenance therapy for GBM patients $(150 \mathrm{mg} /$ $\mathrm{m}^{2}$ ). The mean maximum cross-sectional area was $12.69 \pm$ $2.47 \mathrm{~mm}^{2}$ in control mice, $8.23 \pm 1.68 \mathrm{~mm}^{2}$ in mice treated with $50 \mathrm{mg} / \mathrm{kg}$ of TMZ, and $5.30 \pm 0.65 \mathrm{~mm}^{2}$ in mice treated with $50 \mathrm{mg} / \mathrm{kg}$ of TMZ combined with $15 \mathrm{mg} / \mathrm{kg}$ of riluzole (Fig. 5B and C). The mean tumor volumes were $30.02 \pm 6.99 \mathrm{~mm}^{3}, 15.55 \pm 4.17 \mathrm{~mm}^{3}$, and $9.84 \pm 1.91 \mathrm{~mm}^{3}$ for control, TMZ-treated, and combination-treated mice, respectively (Fig. 5D). TMZ monotherapy did not significantly decrease the mean maximum cross-sectional area of the tumors or the mean tumor volumes in comparison with the control group. However, combinatorial therapy with both TMZ and riluzole significantly suppressed not only the mean maximum cross-sectional area of the tumors, but also the mean tumor volumes in comparison with the control group $(\mathrm{p}<0.05)$. The mean maximum cross-sectional area of the tumors and the mean tumor volumes were slightly but not significantly lower in mice treated with combinatorial therapy than in those treated with TMZ monotherapy.

\section{Discussion}

The present study investigated the effectiveness of combinatorial treatment with riluzole and conventional 

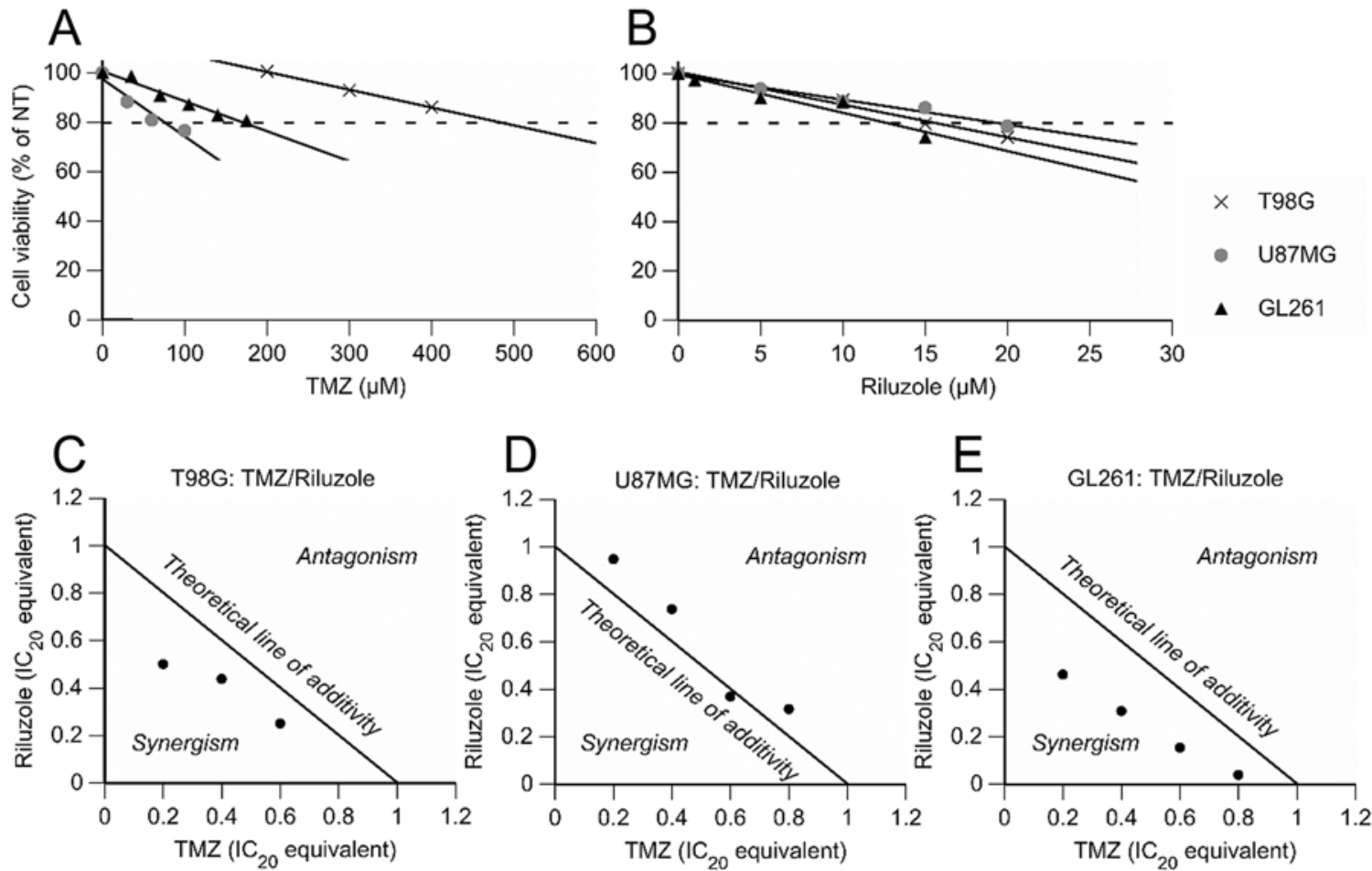

FIG. 3. IC $\mathrm{C}_{20}$ evaluation and isobologram analyses of the effects of different combinations of TMZ and riluzole on the GBM cell lines T98G, U87MG, and GL261. A and B: The 20\% inhibitory concentration $\left(I_{20}\right)$ values for TMZ and riluzole were determined in the T98G, U87MG, and GL261 cell lines through CCK-8 cell viability assays. Herein, the $\mathrm{IC}_{20}$ is defined as the drug concentration that reduces cell viability to $20 \%$ of the no-treatment control cells after 72 hours. In the T98G, U87MG, and GL261 cell lines, the $I C_{20}$ for TMZ was $481 \mu \mathrm{M}, 75 \mu \mathrm{M}$, and $170 \mu \mathrm{M}$, and the $\mathrm{IC}_{20}$ for riluzole was $16 \mu \mathrm{M}, 19 \mu \mathrm{M}$, and $13 \mu \mathrm{M}$, respectively. C-E: Isobologram analyses of the effects of the combined TMZ and riluzole were conducted for T98G (C), U87MG (D), and GL261 (E) cell lines. The "theoretical line of additivity," which connects the $\mathrm{IC}_{20}$ values of the two drugs used in combination therapy, separates the $x-y$ plane into two parts. The points (dots) plotted below, on, or above the line indicate synergism, additivity, or antagonism of the drug combination, respectively. In the T98G (C) and GL261 (E) cell lines, the isobolograms indicated clear synergism at all concentrations tested. In the U87MG cell line (D), the isobologram indicated additivity or antagonism at all concentrations tested.

TMZ in terms of GBM cell growth in vitro and in vivo. The results indicate the following three novel aspects: 1) riluzole synergistically enhanced the antitumor effect of TMZ in MGMT-positive GBM cells; 2) mechanistically, riluzole directly suppressed MGMT expression and suppressed TMZ-induced MGMT upregulation; and 3) synergistic effects of riluzole were observed in a mouse allograft model of MGMT-positive GBM.

Glutamatergic systems potentially play important roles not only in several neurological disorders, including epilepsy, ${ }^{2}$ Alzheimer's disease, ${ }^{32}$ Parkinson's disease, ${ }^{4}$ and amyotrophic lateral sclerosis ${ }^{8}$ but also in tumors such as melanoma and GBM. ${ }^{16,25}$ Recent studies have reported that inhibition of the glutamatergic pathway may be effective for GBM treatment. Riluzole, a selective mGluR1 antagonist, inhibits activation of the PI3K/AKT/mTOR pathway and induces the death of GBM cells. ${ }^{36}$ However, to our knowledge, no studies have investigated the effectiveness of combinatorial treatment of TMZ and riluzole. In the present study, cell viability assays revealed significant synergistic effects between TMZ and riluzole in MGMTpositive GBM cell lines but not in the MGMT-negative GBM cell line. Indeed, in the MGMT-positive cell lines, treatment with riluzole at an ineffective concentration during monotherapy significantly enhanced the effect of TMZ at various clinical concentrations. Furthermore, isobolograms obtained through combinatorial treatment in MGMT-positive GBM cells displayed clear synergism between TMZ and riluzole. These results clearly indicate that riluzole synergistically enhances the antitumor effect of TMZ in MGMT-positive GBM cells.

In MGMT-positive GBM cells, treatment with TMZ upregulates MGMT. ${ }^{17}$ Accordingly, MGMT is a potentially important therapeutic target for GBM therapy. Recent studies have reported that several agents suppress MGMT in GBM cells via various pathways, such as the $\mathrm{Wnt} / \beta$ catenin, Hedgehog/GLI1, and PI3K/AKT/NFKB signaling pathways. ${ }^{3,17,19}$ To our knowledge, previous studies have reported that treatment with interferon- $\beta$ (IFN- $\beta$ ), levetiracetam, valproic acid, bortezomib, and disulfiram downregulate MGMT in GBM cells. ${ }^{5,20,22,24,31}$ In contrast, another study reported that valproic acid upregulates MGMT. ${ }^{5}$ Antagonists of the glutamatergic pathway, including riluzole, have not previously been reported to inhibit MGMT expression in GBM cells. This study is the first to report that riluzole monotherapy downregulates MGMT mRNA in a dose-dependent manner in T98G MGMT-positive GBM cells. As shown in Fig. 4 right, TMZ monotherapy 
TABLE 1. Combination index analysis

\begin{tabular}{|c|c|c|c|c|c|c|}
\hline Cell Lines & $\mathrm{TMZ}(\mu \mathrm{M})$ & Riluzole $(\mu \mathrm{M})$ & $\mathrm{TMZ}_{\mathrm{IC}} \mathrm{C}_{20}$ Equivalent & Riluzole $\mathrm{IC}_{20}$ Equivalent & Combination Index & Judgment \\
\hline \multirow{5}{*}{ T98G: MGMT+ } & 481 & 0 & 1.0000 & 0.0000 & & \\
\hline & 289 & 4 & 0.6000 & 0.2500 & 0.85 & Synergism \\
\hline & 192 & 7 & 0.4000 & 0.4375 & 0.84 & Synergism \\
\hline & 96 & 8 & 0.2000 & 0.5000 & 0.70 & Synergism \\
\hline & 0 & 16 & 0.0000 & 1.0000 & & \\
\hline \multirow{6}{*}{ U87MG: MGMT- } & 75 & 0 & 1.0000 & 0.0000 & & \\
\hline & 60 & 6 & 0.8000 & 0.3157 & 1.12 & Antagonism \\
\hline & 45 & 7 & 0.6000 & 0.3684 & 0.97 & Additivity \\
\hline & 30 & 14 & 0.4000 & 0.7368 & 1.14 & Antagonism \\
\hline & 15 & 18 & 0.2000 & 0.9474 & 1.15 & Antagonism \\
\hline & 0 & 19 & 0.0000 & 1.0000 & & \\
\hline \multirow{6}{*}{ GL261: MGMT+ } & 170 & 0 & 1.0000 & 0.0000 & & \\
\hline & 136 & 0.5 & 0.8000 & 0.0385 & 0.84 & Synergism \\
\hline & 102 & 2 & 0.6000 & 0.1538 & 0.75 & Synergism \\
\hline & 68 & 4 & 0.4000 & 0.3077 & 0.71 & Synergism \\
\hline & 34 & 6 & 0.2000 & 0.4615 & 0.66 & Synergism \\
\hline & 0 & 13 & 0.0000 & 1.0000 & & \\
\hline
\end{tabular}

significantly upregulated MGMT in T98G GBM cells; MGMT was significantly downregulated when cells were treated with riluzol. These results suggest that combinatorial treatment with riluzole and TMZ potentially suppresses TMZ-induced MGMT upregulation in MGMT-positive GBM. Moreover, the concentrations of riluzole used for in vitro combinatorial treatments were within the range of plasma concentrations of healthy volunteers in clinical trials. ${ }^{15}$

A number of clinical trials have investigated the efficacy of several combinatorial therapies with TMZ for GBM treatment, including bevacizumab and IFN- $\beta .{ }^{7,13}$ However, no agents have reportedly prolonged the overall survival of GBM patients receiving combinatorial treatment with TMZ. Thus far, several agents have reportedly exerted combined effects with TMZ in experimental animal models. ${ }^{9,14,35}$ To our knowledge, IFN- $\alpha / \beta$ is the only agent that enhances the effect of TMZ via suppression of MGMT in a mouse model of intracranial GBM..$^{20,26}$ In the present study, we generated a mouse model with a normal immune system using the GL261 MGMT-positive mouse GBM cell line. Compared with control mice, TMZ monotherapy did not significantly suppress tumor size in terms of both maximum cross-sectional area and volume. In contrast, TMZ combined with riluzole significantly suppressed tumor growth in comparison with that seen in control mice.
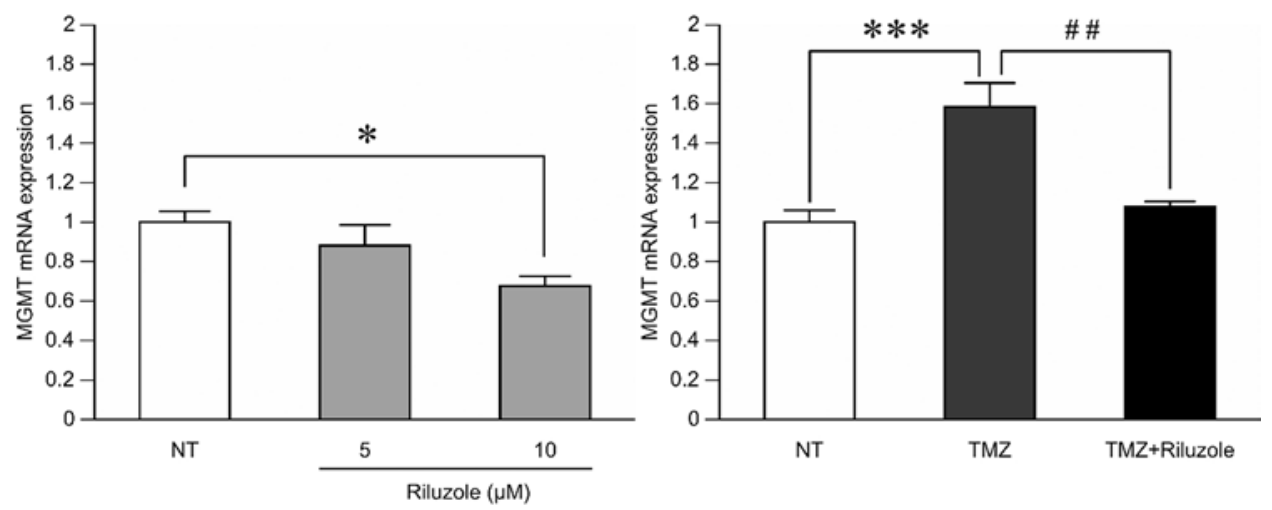

FIG. 4. The effects of riluzole on MGMT mRNA levels in the MGMT-positive GBM cell line T98G. Left: Quantitative RT-PCR (qRT-PCR) analysis was performed after 48 hours of treatment with riluzole at 2 different concentrations ( 5 and $10 \mu \mathrm{M})$. Each column and bar represent the mean \pm SEM ( $n=4$ or 5 ). ${ }^{*} p<0.05$ versus NT (one-way ANOVA followed by Dunnett's test). Right: qRT-PCR analysis after 48 hours of treatment with $300 \mu \mathrm{M}$ of TMZ displayed significant upregulation of MGMT mRNA expression. However, treatment with $5 \mu \mathrm{M}$ of riluzole significantly reduced the TMZ-induced MGMT upregulation. Each column and bar represents the mean \pm SEM $(n=5) .{ }^{* *} p<0.001$ versus NT; \#\#p < 0.01 versus TMZ alone (one-way ANOVA followed by Tukey's test). 
A

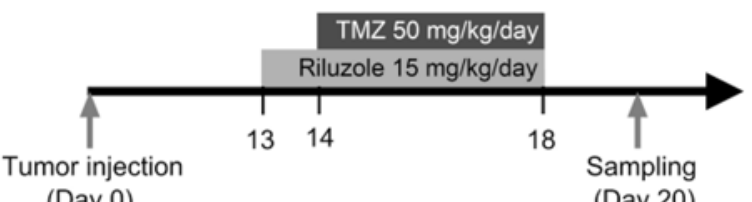

B

NT

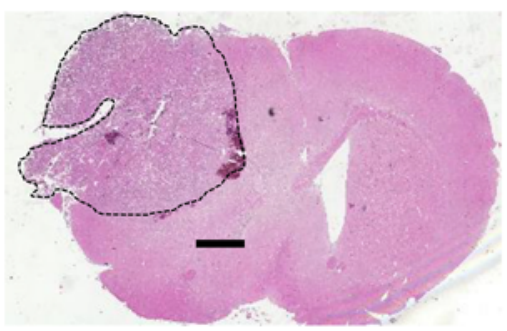

TMZ

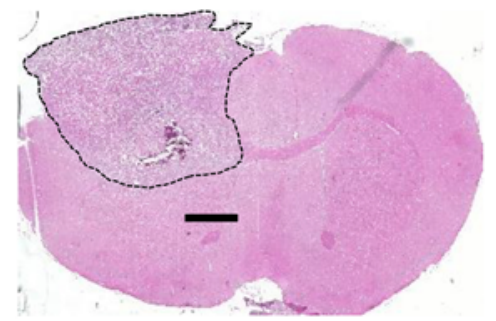

TMZ+Riluzole

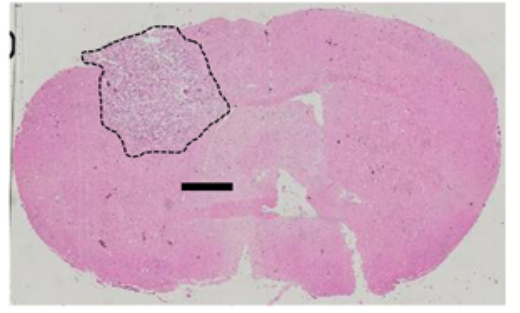

$*$
C

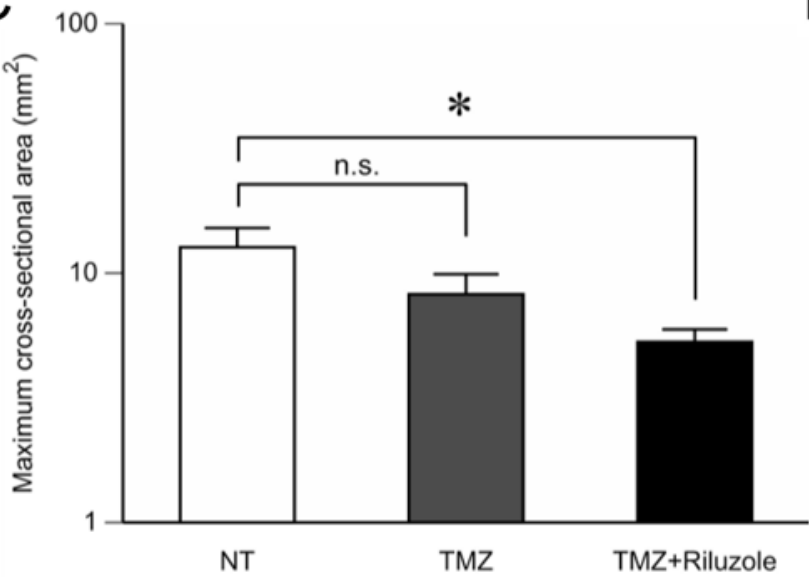

D

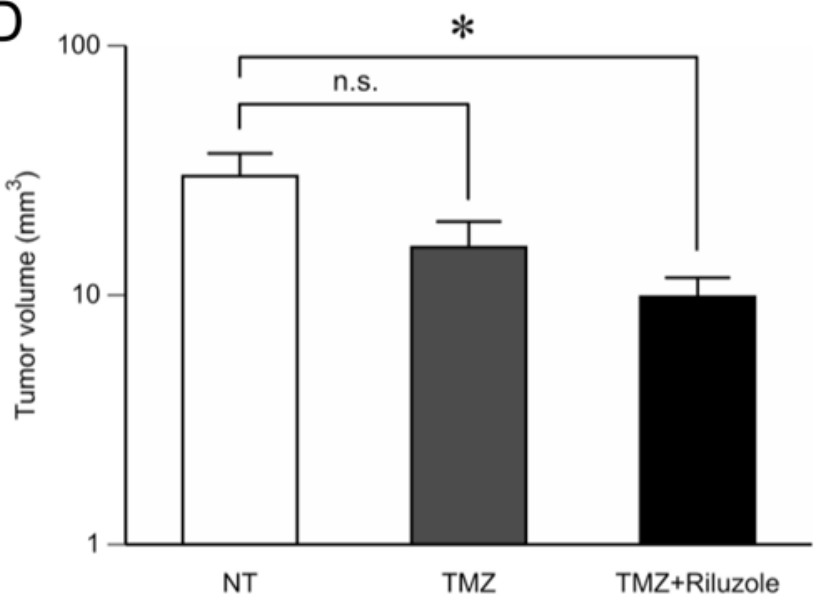

FIG. 5. The effects of combinatorial therapy with TMZ and riluzole on the growth of MGMT-positive GBM in vivo. A: Scheme showing the time point of administration of TMZ and riluzole to mice. B: Photomicrographs showing the $\mathrm{H} \& \mathrm{E}$-stained murine brain coronal sections following treatment of each group. Original magnification $\times 4$. Scale bar $=1 \mathrm{~mm}$. The dashed lines indicate the margins of the tumors. $C$ and $D$ : Bar graphs representing the mean maximum cross-sectional areas $(C)$ and mean tumor volumes (D) of each group. Each column and bar represents the mean \pm SEM $(n=10) ;{ }^{*} p<0.05$ versus NT; $n . s$. = not significant (one-way ANOVA followed by Dunnett's test).

These results suggest that combinatorial treatment with riluzole and TMZ enhances the antitumor effect of TMZ and may improve the prognosis in patients with MGMTpositive GBM via suppression of MGMT. However, clinical trials are required to confirm whether combinatorial treatment with riluzole and TMZ is as effective in human patients as in experimental animal models.

This study, to our knowledge, is the first to report that riluzole enhances the effect of TMZ against MGMT-positive GBM cells via suppression of MGMT expression (Fig. 4). Previous studies have indicated that riluzole exerts its antitumor effects by inhibiting the mGluR1/PI3K/AKT/ mTOR signaling pathway. ${ }^{28,36}$ Other studies have reported that a PI3K inhibitor reduces chemoresistance to TMZ in GBM cells, that another PI3K inhibitor suppresses MGMT expression via inhibition of $\mathrm{PI} 3 \mathrm{~K} / \mathrm{AKT} / \mathrm{NF} \mathrm{KB}$ signaling, and that inhibition of NFKB downregulates MGMT ex- pression..$^{17,27,34}$ These previous studies indicate that riluzole suppresses MGMT expression by inhibiting the mGluR1/ $\mathrm{PI} 3 \mathrm{~K} / \mathrm{AKT} / \mathrm{NF} \mathrm{B}$ signaling pathway. Further studies are required to completely elucidate the effect of riluzole on NFKB signaling.

\section{Conclusions}

This study shows that when administering TMZ monotherapy, MGMT-positive GBM cells displayed MGMT upregulation and chemoresistance to TMZ. Riluzole suppressed the TMZ-induced upregulation of MGMT and enhanced the antitumor effect of TMZ in MGMT-positive GBM in vivo. In conclusion, our results indicate that combinatorial treatment of TMZ/riluzole is a potential therapy for the effective treatment of patients with MGMTpositive GBM. 


\section{References}

1. Azzalin A, Nato G, Parmigiani E, Garello F, Buffo A, Magrassi L: Inhibitors of GLUT/SLC2A enhance the action of BCNU and temozolomide against high-grade gliomas. Neoplasia 19:364-373, 2017

2. Barker-Haliski M, White HS: Glutamatergic mechanisms associated with seizures and epilepsy. Cold Spring Harb Perspect Med 5:a022863, 2015

3. Bi Y, Li H, Yi D, Bai Y, Zhong S, Liu Q, et al: $\beta$-catenin contributes to cordycepin-induced MGMT inhibition and reduction of temozolomide resistance in glioma cells by increasing intracellular reactive oxygen species. Cancer Lett 435:66-79, 2018

4. Blandini F, Porter RH, Greenamyre JT: Glutamate and Parkinson's disease. Mol Neurobiol 12:73-94, 1996

5. Bobustuc GC, Baker CH, Limaye A, Jenkins WD, Pearl G, Avgeropoulos NG, et al: Levetiracetam enhances p53-mediated MGMT inhibition and sensitizes glioblastoma cells to temozolomide. Neuro Oncol 12:917-927, 2010

6. Chahal M, Abdulkarim B, Xu Y, Guiot MC, Easaw JC, Stifani N, et al: $\mathrm{O}(6)-$ Methylguanine-DNA methyltransferase is a novel negative effector of invasion in glioblastoma multiforme. Mol Cancer Ther 11:2440-2450, 2012

7. Chinot OL, Wick W, Mason W, Henriksson R, Saran F, Nishikawa R, et al: Bevacizumab plus radiotherapy-temozolomide for newly diagnosed glioblastoma. N Engl J Med 370:709-722, 2014

8. Corona JC, Tovar-y-Romo LB, Tapia R: Glutamate excitotoxicity and therapeutic targets for amyotrophic lateral sclerosis. Expert Opin Ther Targets 11:1415-1428, 2007

9. Da Ros M, Iorio AL, De Gregorio V, Fantappiè O, Laffi G, de Martino M, et al: Aldoxorubicin and Temozolomide combination in a xenograft mice model of human glioblastoma. Oncotarget 9:34935-34944, 2018

10. de Groot J, Sontheimer H: Glutamate and the biology of gliomas. Glia 59:1181-1189, 2011

11. Doble A: The pharmacology and mechanism of action of riluzole. Neurology 47 (6 Suppl 4):S233-S241, 1996

12. Friedman HS: Temozolomide in early stages of newly diagnosed malignant glioma and neoplastic meningitis. Semin Oncol 27 (3 Suppl 6):35-40, 2000

13. Gilbert MR, Dignam JJ, Armstrong TS, Wefel JS, Blumenthal DT, Vogelbaum MA, et al: A randomized trial of bevacizumab for newly diagnosed glioblastoma. N Engl J Med 370:699-708, 2014

14. Jensen KV, Cseh O, Aman A, Weiss S, Luchman HA: The JAK2/STAT3 inhibitor pacritinib effectively inhibits patientderived GBM brain tumor initiating cells in vitro and when used in combination with temozolomide increases survival in an orthotopic xenograft model. PLoS One 12:e0189670, 2017

15. Le Liboux A, Lefebvre P, Le Roux Y, Truffinet P, Aubeneau M, Kirkesseli S, et al: Single- and multiple-dose pharmacokinetics of riluzole in white subjects. J Clin Pharmacol 37:820-827, 1997

16. Lee HJ, Wall BA, Wangari-Talbot J, Shin SS, Rosenberg S, Chan JL, et al: Glutamatergic pathway targeting in melanoma: single-agent and combinatorial therapies. Clin Cancer Res 17:7080-7092, 2011

17. Li M, Liang RF, Wang X, Mao Q, Liu YH: BKM120 sensitizes C6 glioma cells to temozolomide via suppression of the $\mathrm{PI} 3 \mathrm{~K} / \mathrm{Akt} / \mathrm{NF}-\kappa \mathrm{B} / \mathrm{MGMT}$ signaling pathway. Oncol Lett 14:6597-6603, 2017

18. Liu L, Gerson SL: Targeted modulation of MGMT: clinical implications. Clin Cancer Res 12:328-331, 2006

19. Melamed JR, Morgan JT, Ioele SA, Gleghorn JP, SimsMourtada J, Day ES: Investigating the role of Hedgehog/ GLI1 signaling in glioblastoma cell response to temozolomide. Oncotarget 9:27000-27015, 2018
20. Natsume A, Ishii D, Wakabayashi T, Tsuno T, Hatano H, Mizuno M, et al: IFN- $\beta$ down-regulates the expression of DNA repair gene MGMT and sensitizes resistant glioma cells to temozolomide. Cancer Res 65:7573-7579, 2005

21. Ono Y, Chiba S, Yano H, Nakayama N, Saio M, Tsuruma $\mathrm{K}$, et al: Glycoprotein nonmetastatic melanoma protein B (GPNMB) promotes the progression of brain glioblastoma via $\mathrm{Na}^{+} / \mathrm{K}^{+}$-ATPase. Biochem Biophys Res Commun 481:712,2016

22. Paranjpe A, Zhang R, Ali-Osman F, Bobustuc GC, Srivenugopal KS: Disulfiram is a direct and potent inhibitor of human $\mathrm{O}^{6}$-methylguanine-DNA methyltransferase (MGMT) in brain tumor cells and mouse brain and markedly increases the alkylating DNA damage. Carcinogenesis 35:692-702, 2014

23. Quinn JA, Jiang SX, Reardon DA, Desjardins A, Vredenburgh JJ, Rich JN, et al: Phase II trial of temozolomide plus $\mathrm{O}^{6}$-benzylguanine in adults with recurrent, temozolomide-resistant malignant glioma. J Clin Oncol 27:1262-1267, 2009

24. Ryu CH, Yoon WS, Park KY, Kim SM, Lim JY, Woo JS, et al: Valproic acid downregulates the expression of MGMT and sensitizes temozolomide-resistant glioma cells. J Biomed Biotechnol 2012:987495, 2012

25. Rzeski W, Ikonomidou C, Turski L: Glutamate antagonists limit tumor growth. Biochem Pharmacol 64:1195-1200, 2002

26. Shen D, Guo CC, Wang J, Qiu ZK, Sai K, Yang QY, et al: Interferon- $\alpha / \beta$ enhances temozolomide activity against MGMT-positive glioma stem-like cells. Oncol Rep 34:27152721, 2015

27. Shi F, Guo H, Zhang R, Liu H, Wu L, Wu Q, et al: The PI3K inhibitor GDC-0941 enhances radiosensitization and reduces chemoresistance to temozolomide in GBM cell lines. Neuroscience 346:298-308, 2017

28. Sperling S, Aung T, Martin S, Rohde V, Ninkovic M: Riluzole: a potential therapeutic intervention in human brain tumor stem-like cells. Oncotarget 8:96697-96709, 2017

29. Stupp R, Mason WP, van den Bent MJ, Weller M, Fisher $\mathrm{B}$, Taphoorn MJ, et al: Radiotherapy plus concomitant and adjuvant temozolomide for glioblastoma. $\mathbf{N}$ Engl J Med 352:987-996, 2005

30. Viel T, Monfared P, Schelhaas S, Fricke IB, Kuhlmann MT, Fraefel C, et al: Optimizing glioblastoma temozolomide chemotherapy employing lentiviral-based anti-MGMT shRNA technology. Mol Ther 21:570-579, 2013

31. Vlachostergios PJ, Hatzidaki E, Stathakis NE, Koukoulis GK, Papandreou CN: Bortezomib downregulates MGMT expression in T98G glioblastoma cells. Cell Mol Neurobiol 33:313-318, 2013

32. Wang R, Reddy PH: Role of glutamate and NMDA receptors in Alzheimer's disease. J Alzheimers Dis 57:1041-1048, 2017

33. Yohay K, Tyler B, Weaver KD, Pardo AC, Gincel D, Blakeley $\mathrm{J}$, et al: Efficacy of local polymer-based and systemic delivery of the anti-glutamatergic agents riluzole and memantine in rat glioma models. J Neurosurg 120:854-863, 2014

34. Yu Z, Chen Y, Wang S, Li P, Zhou G, Yuan Y: Inhibition of $\mathrm{NF}-\kappa \mathrm{B}$ results in anti-glioma activity and reduces temozolomide-induced chemoresistance by down-regulating MGMT gene expression. Cancer Lett 428:77-89, 2018

35. Yu Z, Zhao G, Xie G, Zhao L, Chen Y, Yu H, et al: Metformin and temozolomide act synergistically to inhibit growth of glioma cells and glioma stem cells in vitro and in vivo. Oncotarget 6:32930-32943, 2015

36. Zhang C, Yuan XR, Li HY, Zhao ZJ, Liao YW, Wang XY, et al: Anti-cancer effect of metabotropic glutamate receptor 1 inhibition in human glioma U87 cells: involvement of PI3K/ Akt/mTOR pathway. Cell Physiol Biochem 35:419-432, 2015 
37. Zhu T, Shen Y, Tang Q, Chen L, Gao H, Zhu J: BCNU/PLGA microspheres: a promising strategy for the treatment of gliomas in mice. Chin J Cancer Res 26:81-88, 2014

\section{Disclosures}

The authors report no conflict of interest concerning the materials or methods used in this study or the findings specified in this paper.

\section{Author Contributions}

Conception and design: Hara, Yamada, Tsuji, Nakamura, Yano. Acquisition of data: Yamada, Tsuji. Analysis and interpretation of data: Yamada, Tsuji. Drafting the article: Yamada. Critically revising the article: Hara, Nakamura, Egashira, Shimazawa,
Nakayama, Yano, Iwama. Reviewed submitted version of manuscript: Hara, Tsuji, Nakamura, Egashira, Shimazawa, Nakayama, Yano, Iwama. Approved the final version of the manuscript on behalf of all authors: Hara. Statistical analysis: Hara, Yamada, Tsuji, Shimazawa. Administrative/technical/material support: Hara, Egashira. Study supervision: Hara, Nakamura, Shimazawa, Iwama.

\section{Correspondence}

Hideaki Hara: Gifu Pharmaceutical University, Gifu, Japan. hidehara@gifu-pu.ac.jp. 